\title{
Crystalline Thin Films of Silica: Modelling, Structure and Energetics.
}

\author{
Mark Wilson, Harry Jenkins \\ E-mail: mark.wilson@chem.ox.ac.uk \\ Department of Chemistry, Physical and Theoretical Chemistry Laboratory, \\ University of Oxford, South Parks Road, Oxford OX1 3QZ, U.K.
}




\begin{abstract}
The static structural and energetic properties of thin crystalline films ( two dimensional bilayers) of silica, $\mathrm{SiO}_{2}$, are modelled. Two potential models are considered in which the key interactions are described by purely harmonic terms and more complex electrostatic terms, respectively. The relative energetic stability of two potential crystalline forms, which represent alternative ways of tiling two dimensional space, is discussed. Coherent and incoherent distortions are introduced to the simulated crystals and their effects considered in terms of the ring structure formed by the $\mathrm{Si}$ atoms. The spatial relationship between distorted rings is analysed. An experimentally-observed single crystalline configuration is considered for comparison throughout.
\end{abstract}

\title{
1. Introduction.
}

Silica, $\mathrm{SiO}_{2}$, represents an archetypal network-forming material. At ambient pressure both the crystalline and amorphous structures can reasonably be considered as constructed from corner-sharing $\mathrm{SiO}_{4}$ tetrahedral coordination polyhedra (CP) [1, 2] . The network nature of the underlying structure may lead to significant ordering on length-scales beyond the cation/anion short-range ordering imposed by electrostatics (relative atom electronegativities) $[3,4,5]$. The nature and origin of any long-range ordering may be deduced directly from experimental diffraction studies [6]. However, the pair distribution functions typically generated by these studies, although highly informative, can be ambiguous. Signatures of long-range order are often "hidden" at long separations with a range of potential structural motifs able to give rise to nearidentical pair distribution functions. Potential data manipulation issues, such as the imposition of fourier transform truncation errors, further complicates the interpretation of these functions.

Recently developed synthetic pathways have allowed thin films of $\mathrm{SiO}_{2}$ to be deposited on either metallic $[7,8,9,10,11,12,13,14,15,16]$ or graphitic [17] substrates, whilst advances in imaging techniques now allow for true atomic resolution of the surface structure. Some of the thinnest films deposited are interpreted as having structures based on bilayers of corner-sharing $\mathrm{SiO}_{4} \mathrm{CP}$ in which all of the $\mathrm{Si}$ and $\mathrm{O}$ atoms obtain their full (four- and two- respectively) coordination numbers. Amorphous and crystalline films have been grown with both states characterised by the presence of a mirror plane (which houses a layer of $\mathrm{O}$ atoms which act as bridges between the two monolayers $[18,19])$. Critically, the pseudo-two dimensional nature of these systems allows the ring structures to be directly observed and hence offers a potentially unique insight into the origin of any ordering on different length-scales. For example, the crystalline system can be considered as constructed exclusively from a percolating net of six-membered (Si-SiSi...) rings, whilst the amorphous systems are constructed from a distribution of 4to 10 -membered rings $[20,21]$. However, the experimental information, whilst groundbreaking, is naturally imperfect. The location of each atom has associated with it a natural uncertainty (which translates into an uncertainty in atom-atom separations or, 
equivalently, in bond lengths). Furthermore, it is common for the location of one atom type to be resolved over the other meaning that chemical intuition must be applied to place the remaining atoms (for example, placing the $\mathrm{O}$ atoms halfway between neighbouring pairs of $\mathrm{Si}$ atoms). In addition, there may be systematic errors in that there may not be a clear length-scale metric, potentially resulting in a systematic error in all observed bond lengths.

Simulation models may offer a route for further interpretation and potential refinement of this important experimental information. Simulation models can greatly aid the interpretation of these data as the atom positions are known unequivocally. As a result, information such as the ring statistics, which is in many ways a natural language for discussing network structure [22, 3, 23], is directly accessible. In the three dimensional glasses more commonly studied, however, the ring size distribution is not directly accessible although models may be developed which connect the mean ring size to the mean nearest-neighbour Si-O coordination number (which may be measured) [3]. However, the success of the simulation models relies heavily on the accuracy of the potentials employed and their transferability both in terms of chemical composition and pressure-temperature state point.

In this paper we shall focus on crystalline bilayer structures as potentially the most readily understandable. The crystalline configuration may be the clearest way of discussing the experimental data as it should be relatively unambiguous. However, there may be experimental uncertainty and specific unknown distortions. for example, the crystalline section observed directly (as part of a crystalline/amorphous interface $[24,25])$ shows characteristic distortions in the rings away from an ideal percolating array of six-membered rings. A key question, therefore, is to identify the potential origins of these distortions. The simplest explanation would be that the observed distortions are real, arising from stress fields resulting from interactions with the underlying substrate on which the bilayer is grown or resulting from the presence of the interface, or even resulting from interactions with other nearby deposited material. Alternatively the distortions may reflect the underlying experimental uncertainty in the atom coordinates. As a result, an investigation into any spatial correlations between distorted rings may be significant with a coherent distribution of distortions more likely indicative of a specific interaction than a random distribution. To investigate these possibilities we employ simulation models of varying complexity to establish the factors which may influence and control the bilayer crystal structure. The use of simulation models allows reasonable statistics to be generated and, therefore, will hopefully augment the highly detailed experimental information at present "limited" to a single configuration.

\section{The Experimental Information.}

Figure 1 shows the configuration extracted from experiment [24] highlighting the Si atoms only. The system shows a clear interface between a crystalline region 
(characterised by the presence of six-membered rings only $\ddagger$ and an amorphous region (characterised by a mixture of 4 - to 10 -membered rings). The configuration is divided (by eye) as shown to generate a purely crystalline configuration (containing $423 \mathrm{Si}$ atoms) and a smaller amorphous configuration (containing $294 \mathrm{Si}$ atoms).

Figure 2(a) shows the radial distribution functions (rdfs) determined between $\mathrm{Si}$ atom pairs calculated for both the crystalline and amorphous regions with the $\mathrm{Si}$ atoms are assumed to sit in a single plane. The widths of the first peaks are similar for both crystalline and amorphous regions $\left(\Delta r_{F W H M} \sim 0.60 \AA\right)$. Clear differences in the disordered and crystalline distributions are observed at distances beyond those associated with the nearest-neighbours, indicative of the distribution of ring sizes present in the former. To place these distributions into some sort of context, figure 2(b) shows a comparison of the rdfs generated here with those generated for bulk glassy $\mathrm{SiO}_{2}$ at ambient pressure using an established potential model (see below) [26, 3]. Two comparisons are highlighted. In the first the rdfs generated here are compared with the bulk $g_{S i S i}(r)$, whilst in the second they are compared to bulk $g_{S i O}\left(r^{\prime}\right)$ in which the Si-O length-scale has been doubled. The simulated bulk $g_{S i S i}(r)$ appears significantly narrower with $\Delta r_{F W H M} \sim 0.27 \AA . g_{S i O}\left(r^{\prime}\right)$ highlights the theoretical maximum of the $\mathrm{Si}-\mathrm{Si}$ separations in the sense that, if the Si-O-Si linkages were linear, then $r_{\text {SiSi }}=2 r_{\text {SiO }}$. The relationships between $g_{S i O}\left(r^{\prime}\right)$ and $g_{S i S i}(r)$ for the bulk (in which the latter appears at a shorter length-scale than the former) reflects the non-linear nature of the Si-O-Si bridges.

The implication of the raw data presented in figures $2(\mathrm{a})$ and (b) is that the distribution of nearest-neighbour Si-Si separations appears relatively broad whilst a significant proportion of these separations appear "long" in term of their relationship with the expected nearest-neighbour Si-O separations. Figure 3 shows the crystalline configuration with a range of different $\mathrm{Si}-\mathrm{Si}$ distances selected. Around $4 \%$ of the $\mathrm{Si}-\mathrm{Si}$ nearest-neighbour separations are greater than $3.5 \AA$ rising to $\sim 57 \%>3.0 \AA$ and $\sim 98 \%$ $>2.5 \AA$ (also highlighted by the running coordination number shown in figure $2(\mathrm{a})$ ). To attempt to further break down the experimental information figures 2(c) and (d) show the distributions and cumulative distributions of the nearest-neighbour Si-Si separations divided into contributions from Si-Si pairs approximately perpendicular to the original crystal/amorphous interface and those not, here meaning Si-Si pairs at $\sim 30^{\circ}$ to the original interface (see figure 1). The mean nearest-neighbour Si-Si separation from atom pairs perpendicular to the original interface appear $\sim 6.5 \%$ shorter than for those with a significant separation component parallel to the interface. A purely crystalline sample would, with no external influences, be expected to show no difference in these respective bond lengths. The difference here may reflect the role of the interface (although the sample size is too small to determine if any effect diminishes with distance away from the interface) or the role of the substrate or surrounding material.

Figure 2(e) shows the corresponding Si-Si-Si bond angle distributions. The $\ddagger$ An $n$-membered ring here refers to the number of Si atoms in the ring. 
respective peak widths are $\Delta \theta_{F W H M} \simeq 20^{\circ}$ and $\Delta \theta_{F W H M} \simeq 30^{\circ}$ for the crystalline and amorphous regions. The broader distribution for the amorphous configuration again reflects the broader distribution of ring sizes compared with the crystal. Again, the corresponding ambient pressure bulk distribution is shown for comparison. The bulk distribution is significantly broader $\left(\Delta \theta_{F W H M} \simeq 43^{\circ}\right)$ with a peak at $\theta \sim 109^{\circ}$ reflecting the underlying three-dimensional tetrahedron-based morphology, compared with the pseudo-two dimensional morphology displayed by the bilayers (reflected in the peak positions of $\theta \sim 120^{\circ}$ ). In general, therefore, the Si-Si distributions appear relatively narrow for the bulk configurations when compared to the bilayers whilst the Si-Si-Si distributions display the opposite trend.

\section{Potential Models.}

As discussed previously for amorphous systems [18] two forms of potential model are considered. In the first harmonic springs connect the four nearest-neighbour Si-O and six nearest-neighbour O-O atoms in each tetrahedron with the O-O and $\mathrm{Si}-\mathrm{O}$ equilibrium bond lengths chosen so as to impose ideal tetrahedra. The spring force constants are taken as one for both the $\mathrm{Si}-\mathrm{O}$ and $\mathrm{O}-\mathrm{O}$ pairs as the detail of these interactions is only significant in allowing for a relatively rapid energy minimization which remaining commensurate with both the atomic masses and the molecular dynamics time step. The harmonic potential may be augmented with a (purely repulsive) potential which acts between pairs of Si atoms only. The chosen form is a shifted 24-12 potential,

$$
U(r)=4 \epsilon\left\{\left(\frac{\sigma}{r}\right)^{24}-\left(\frac{\sigma}{r}\right)^{12}\right\}+\epsilon,
$$

where $\sigma$ is the atom diameter and $\epsilon$ is the well-depth of the (unshifted) potential. The potential is cut-off at the minimum $\left[r_{\text {min }}=(2)^{1 / 12} \sigma\right]$ ensuring continuity in both energy and force as a function of $r$. The parameter $\epsilon$ is fixed (at $\epsilon / k_{B}=10 \mathrm{~K}$ ) whilst $\sigma$ can be varied. Although the repulsive potential acts between Si atom pairs only, it is designed to mimic repulsion between tetrahedra (as the centre of which sit the Si atoms).

In the second form a potential model is applied in which pairwise-additive forms (including ion-based charge-charge electrostatic interactions) are augmented with a description of (many-body) polarization effects [26, 27], termed the TS potential.

\section{Results and Discussion.}

Our strategy is to consider first the idealised crystal structures and then consider how specific distortions may affect their properties.

\subsection{Ideal Crystals.}

Our first calculation is to establish the energy as a function of area for potential bilayer crystal structures. In addition to the six-ring only crystal bilayer we consider an 
alternative based on a percolating network of alternating four- and eight-membered rings (here termed the $4 / 8$ crystal bilayer). Both structures are shown in figure 4(a). The 4/8 crystal structure is considered firstly as an example of an alternative tiling of the pseudo two dimensional space and secondly as it has been considered for the related graphene-like systems $[28,29,30]$ for which clusters of such ring environments have been observed experimentally $[31,32]$. Figure 4(b) shows the energy $v$. area curves for the two bilayer crystals generated using a TS potential. Each point obtained using a steepest descent energy minimisation at fixed area. The energy minima for the respective sixand 4/8-ring bilayers appear at $A=6.20 \AA^{2} /$ molecule $\S$ and $A=6.84 \AA^{2} /$ molecule $\left(\equiv 16.1\right.$ and 14.6 molecules $/ \mathrm{nm}^{2}$ ) with an energy difference between the minima of $\Delta U \sim 12.8 \mathrm{kJmol}^{-1}$. The energy minimum for the $4 / 8$ structure lies are higher area than that form the 6-ring structure, implying the existence of a "region of stability" for $A \geq 6.7 \AA^{2} /$ molecule for the $4 / 8$ structure which could, in theory, be accessed, perhaps by deposition on a suitable surface or growth under stressed conditions.

\subsection{Distorted Crystals.}

4.2.1. Coherent Distortions. The six-ring bilayer crystal may exhibit a coherent distortion of the type shown schematically in figure 5(a). In this distortion the tetrahedra rotate about the $\mathrm{Si}-\mathrm{O}-\mathrm{Si}$ bridge between the two monolayers forming the bilayer. The distortion results in a systematic reduction of the apparent area occupied by rings defined in terms of the Si atoms only as the nearest-neighbour Si-Si separations are reduced as the Si-O-Si bond angles become more acute. Figure 5(b), for example, highlights the ideal distortion to the crystal for relatively small and large Si-O-Si bond angle distortions.

Figure 6 shows the energy of the ideal six-ring bilayer crystal as a function of the reduced area, $A^{*}$, imposing the coherent distortion as shown in figure 5 . The energies are calculated using both the harmonic potential (varying the inter-tetrahedral repulsion through changing the effective diameter $\sigma$ in equation 1) and the TS potential. For the harmonic potential a reduced area of $A^{*}=1$ corresponds to the highest area which can be supported whilst maintaining undistorted $\mathrm{SiO}_{4}$ tetrahedra, whilst for the TS potential $A^{*}=1$ is defined as the location of the energy minimum in figure $4(\mathrm{~b})$. With no intertetrahedral repulsion the harmonic energy can be driven to zero for $A^{*} \leq 1$ (which defines an upper bound on the area, $A_{u}$ ). The Si-O-Si bond angle for the tetrahedra linked in the bilayer plane (i.e. not the triplets linking the upper and lower monolayers) are shown in the inset to figure 6 . For $A^{*} \leq 1$ the angle follows a simple geometric relationship as $A$ varies whilst, for $A^{*} \geq 1$, the change in angle as $A$ increases reflects the distortions of the tetrahedra themselves. Above $A_{u}$ the Si-O and/or O-O bonds must become stretched whilst below this area the coherent twist of the tetrahedra about the Si-O-Si triplets allows the tetrahedra to remain ideal (undistorted) whilst reducing the overall system area. The introduction of an inter-tetrahedral repulsion defines a lower bound on the

$\S$ This includes both layers 
area, $A_{l}$, below which the distortion is so large as to introduce significant overlap in neighbouring tetrahedra. As the magnitude of the repulsion is increases (increasing $\sigma$ ) the lower bound moves to higher area and so the effective flexibility window [33] defined by $A_{u}-A_{l}$, narrows. Above $\sigma / r_{S i O} \sim 1.78$ (a geometric constraint) the energy can no longer be driven to zero as the nearest-neighbour tetrahedra overlap in the undistorted crystal.

Figure 6 also shown the energy obtained using the TS potential (shown as the energy relative to the energy minimum). As previously observed for the amorphous configurations, the use of a more realistic potential acts to "select" a single density from within the window [18]. For amorphous configurations the precise density selected depends upon the ring statistics and, most likely, the distribution of those rings [18]. The density selected corresponds to a reduced area of $A^{*} \sim 0.95$ indicating limited Si-O-Si distortions to be energetically favourable over the undistorted crystal used to generate figure $4(\mathrm{~b})$.

4.2.2. Incoherent Distortions. Considering again the distortions shown in figure 5 . Each $\mathrm{O}$ atom in a given Si-O-Si triplet can effectively translate in one of two equivalent directions perpendicular to the vector joining the two Si atoms. The ideal distortions shown in figure 5 arise from coherent motion across the whole (periodically-repeated) cell. Figure 7 shows the effect of heating the bilayer (described by the full TS potential) and then quenching to low temperature. Under these conditions domains appear in which the $\mathrm{O}$ atoms distort along alternative directions in different regions of the cell. These displacements can be different in the upper and lower layers (here affecting $~ 13 \%$ of the rings) of the bilayer (although the Si sublattice retains the "mirror image" symmetry). The figure also shows how the distortions in the $\mathrm{O}$ sublattice translate into distortions in the underlying Si-atom hexagonal lattice. The distortions appear characteristic of those observed from experiment (figure 1). Figure 4(b) shows the energies as a function of area for the fully relaxed configurations.

Figure 8(a) shows the distribution of ring areas (determined for rings defined by the Si atoms only and projected into the major plane) for the configurations obtained from the heating/cooling cycles at four reduced areas $\left(A^{*}=0.95,0.90,0.86\right.$ and 0.77$)$. At $A^{*}=0.95$ the distribution is relatively narrow reflecting the near-linear Si-O-Si bond angles corresponding to near-ideal hexagons. As the area falls the Si-O-Si bond angle distributions become broader, mirrored in the broader distribution of hexagon areas. The figure also shows the distribution of areas for the experimental crystal configuration (figure 1). The experimental distribution appears both relatively broad and is centred at relatively high area with respect to the simulation results. Figure 8(b) shows the mean ring areas, normalised by the respective ideal areas of the undistorted rings, as a function of the mean nearest-neighbour Si-Si separation, $r_{S i S i}$. For the harmonic potential with the ideal coherent distortion imposed (figure 5) $A / A_{\text {ideal }}=1$ reflecting the symmetry of this distortion which allows $r_{S i S i}$ to fall whilst retaining the ideal hexagonal geometry. For the heating/cooling cycles, for both the harmonic and TS potentials, $A / A_{\text {ideal }}$ falls 
below one as $r_{S i S i}$ is lowered reflecting the presence of more distorted hexagons. The figure also shows the single point obtained from analysis of the experimental crystalline configuration. A scaling of $\sim 0.94$ in the length-scale maps the experimental datum onto the TS model curve, the corresponding scaling for the area distribution being shown in figure $8(\mathrm{a})$.

To attempt to elucidate any spatial relationship between rings of different size and/or shape we divide the area distributions (shown in figure 8(a)) in two, labelling hexagons in the lower quartile of the distribution "1" and all others "2". Pair distribution functions are then constructed defined in terms of the distances between the centres of mass of the respective rings, $r_{C M}$. For an ideal network of percolated hexagons the centres of mass define an ideal close-packed network (there is a dual relationship [34]). Using the centres of mass of the rings defines three functions, $g_{\alpha \beta}\left(r_{C M}\right)[\{\alpha \beta=$ $11,12,22\}$ ], which contain information regarding the spatial relationships between highly distorted (low area) rings and the more ideal (larger) species. The main panel of figure 9 shows the first peaks in these three functions at four different areas $\left(A^{*}=0.81,0.90,0.94\right.$ and 10 respectively). As the area increases the width of the first peaks decrease reflecting the more ideal geometry of the hexagons adopted. These functions may be integrated to generate coordination numbers which may then be compared with those expected for a random distribution in a two-dimensional close-packed lattice of stoichiometry $\mathrm{AB}_{3}$. We define the difference $\Delta n=\bar{n}-n_{r n d}$, where $\bar{n}$ is the coordination number obtained from the pair distribution functions and $n_{r n d}$ is that expected for a random distribution. The inset to figure 9 shows the coordination number differences, $\Delta n$, determined over a range of areas. $\Delta n$ tends to zero for all three spatial correlations as the area gets smaller. As the area increases $\Delta n$ tends to a non-zero value up to the tensile limit ( $\left.A^{*} \sim 1.04\right)$. This is indicative of an effective de-mixing ("phase separation") in which the relatively distorted rings preferentially sit near other distorted rings whilst more ideal rings sit near other near-ideal rings. The non-zero limits as $A^{*}$ reaches the system tensile limit reflects the system stress being concentrated around specific distortions rather than being delocalised throughout the system.

Figure 10 shows the experimental configuration with the lowest quartile of ring areas highlighted. The lowest area (most distorted) rings appear to show a tendency to form chains with other low area rings consistent with ordering on multiple length-scales. However, whilst these correlations may appear clear, the system size is necessarily small and so definitive conclusions are difficult to draw. Future work will focus on developing methods to effectively generate multiple large-scale configurations which will enable a more effective statistical study [35].

\subsection{Comparison to Previous Work and Outlook.}

A primary aim in the current work is to help understand and interpret experimental observation, in particular the potential origin of observed distortions. We propose that the observed distortions can be rationalised by relatively energetically facile distortions 
of the linked tetrahedra. Alternatively, the configurations themselves may be subject to significant internal stresses. In reference [36] density functional theory is applied to consider the relative energetics of bilayers constructed from different ring motifs. The array of percolating hexagons is found to be the most energetically-favourable configuration studied (and relatively close in energy to the bulk $\alpha$-quartz crystal structure) whilst a range of different ring motifs show relatively small energy differences $\left(<10 \mathrm{kJmol}^{-1}\right)$. For example, the $4 / 8$ ring system (figure 4 ) is found to be $\sim 4 \mathrm{kJmol}{ }^{-1}$ less favourable (compared with $\sim 13 \mathrm{kJmol}^{-1}$ found here). The relatively small energetic differences observed for the different ring topologies is unsurprising given the known behaviour of silicates and related aluminosilicates which form a wide range of zeolitic structures within a narrow energy range (in the so-called the "flexibility window" [33]). In the same work the potential effect of relaxing the internal stress is also discussed and linked to the experimental observation that ring "defects" (here meaning non-sixmembered rings) are observed as clusters [36]. It is, however, possible that such strain effects are more acute in the relatively small system sizes necessarily studied when applying DFT. Furthermore, the presence of, for example, a large ring will necessarily favour the formation of a neighbouring small ring (an Aboav-Weaire law - see ref. [37] and references therein).

Recent work has shown $\mathrm{GeO}_{2}$ may be similarly grown as thin films [38], although these appear more difficult to deposit than the corresponding silica and aluminosilicates. Potential analogues of the silica ring topologies for $\mathrm{GeO}_{2}$ are discussed in reference [36], concluding that the germania structures are energetically less favourable than their bulk counterparts when compared with the silica analogues. The rationale is relatively simple as the bilayers require the stabilisation of a near-linear Si-O-Si triplet (joining the two mirror layers). Such near-linear triplets will be less stable for $\mathrm{GeO}_{2}$ than for $\mathrm{SiO}_{2}$ as evidenced from $a b$ initio calculations [39] and the bulk glass bond angles (of $\sim 130^{\circ}$ and $\sim 145^{\circ}$ respectively) [40]. The implication is that bilayer analogues of $\mathrm{SiO}_{2}$ may be formed for systems of the same stoichiometry with relatively obtuse M-O-M bond angles but more difficult to form for systems with more acute angles (see ref. [29] for a discussion of the bond angles in related systems). Alternatively, mixtures of cations may stabilise the required near-linear angles [36]. The coherent distortions considered here required the stabilisation of both relatively acute bond angles (for the distortions in the plain of the bilayer) and near-linear bond angles to join the two monolayers.

\section{Conclusions.}

In this paper some basic structural properties of pseudo-two dimensional crystalline bilayers of $\mathrm{SiO}_{2}$ have been investigated. A coherent distortion, in which the tetrahedra are twisted around the inter-layer Si-O-Si links, allows the apparent ring areas (defined by the Si atoms only) to systematically vary and may be associated with an applied compressive strain. Incoherent distortions, in which domains of linked tetrahedra distort along different, yet equivalent, pathways, lead to distortions of the ring structure 
analogous to those observed experimentally.

\section{Acknowledgements.}

The authors are very grateful to the Fritz-Haber-Institute in Berlin for useful discussions and their great hospitality. 
[1] A.F.Wells. Structural Inorganic chemistry. Clarendon, 1984.

[2] U. Mueller. Inorganic Structural Chemistry. Wiley, 1993.

[3] A.Zeidler, K.Wezka, D.A.J.Whittaker, P.S.Salmon, S.Klotz, H.E.Fischer, M.C.Wilding, C.L.Bull, M.G.Tucker, and M.Wilson. Phys. Rev. Lett., 113:135501, 2014.

[4] A.Zeidler and P.S.Salmon. in Molecular Dynamics Dimulations of Disordered Materials. Springer, 2015.

[5] F.A.Cotton, G.Wilkinson, C.A.Murillo, and M.Bochmann. Advanced Inorganic Chemistry. WileyBlackwell, 1999.

[6] H.E.Fischer, A.C.Barnes, and P.S.Salmon. Rep. Prog. Phys., 69:233, 2006.

[7] J.Weissenrieder, S.Kaya, J-L.Lu, H-J.Gao, S.Shaikhutdinov, H-J.Freund, M.Sierka, T.K.Todorova, and J.Sauer. Atomic structure of a thin silica film on a mo(112) substrate: A two-dimensional network of $\mathrm{sio}_{2}$ tetrahedra. Phys. Rev. Lett., 95:076103, 2005.

[8] L.Lichtenstein, C. Buechner, B.Yang, S.Shaikhutdinov, M.Heyde, M.Sierka, R.Wlodarczyk, J.Sauer, and H-J Freund. The atomic structure of a metal-supported vitreous thin silica film. Angew. Chem. Int. Ed., 51:404, 2012.

[9] M.Heyde, S.Shaikhutdinov, and J.-J Freund. Two-dimensional silica: Crystalline and vitreous. Chem. Phys. Lett., 550:1, 2012.

[10] D.Loeffler, J.J.Uhliruch, M.Baron, B.Yang, X.Yu, L.Lichtenstein, L.Heinke, C.Buechner, M.Heyde, S.Shaikhutdinov, H-J.Freund, R.Wlodarczyk, M.Sierka, and J.Sauer. Growth and structure of crystalline silica sheet on ru(0001). Phys. Rev. Lett., 105:146104, 2010.

[11] E.I.Altman, J.Gotzen, N.Samudrala, and U.D.Schwarz. J. Phys. Chem. C, 117:26144, 2013.

[12] E.I.Altman and U.D.Schwarz. Adv. Mat. Int., 1:1400108, 2014.

[13] C. Buechner and M.Heyde. Two-dimensional silica opens new perspectives. Prog. Surf. Sci., 92:341-374, 2017.

[14] C. Buechner, Z-J.Wang, K.M.Burson, M-G. Willinger, , M.Heyde, R.Schloegl, and H-J Freund. A large-area transferable wide band gap 2d silicon dioxide layer. ACS Nano., 10:7982-7989, 2016.

[15] C. Buechner, L.Liu, S.Stuckenholz, K.M.Burson, L. Lichtenstein, M.Heyde, H-J.Gao, and H-J Freund. Building block analysis of $2 \mathrm{~d}$ amorphous networks reveals medium range correlation. J. Non-Crys. Solids, 435:40-47, 2016.

[16] K.M.Burson, P.Schlexer, C. Buechner, L. Lichtenstein, M.Heyde, and H-J Freund. Characterizing crystalline-vitreous structures: From atomically resolved silica to macroscopic bubble rafts. $J$. Chem. Educ., 92:1896-1902, 2015.

[17] P.Y.Huang, S.Kurasch, A.Srivastava, V.Skakalova, J.Kotakoski, A.V.Krasheninnikov, R.Hovden, Q.Mao, J.C.Meyer, J.Smet, D.A.Muller, and U.Kaiser. Nano. Lett., 12:1081, 2012.

[18] M.Wilson, A.Kumar, D.Sherrington, and M.F.Thorpe. Phys. Rev. B, 87:214108, 2013.

[19] M. Sadjadi, B. Bhattarai, D.A. Drabold, M.F. Thorpe, and M. Wilson. Refining glass structure in two dimensions. Phys. Rev. B, 96:201405(R), 2017.

[20] A.Kumar, D.Sherrington, M.Wilson, and M.F.Thorpe. Ring statistics of silica bilayers. J. Phys.: Condens. Mat., 26:395401, 2014.

[21] M. Sadjadi and M.F. Thorpe. Ring correlations in random networks. Phys. Rev. E, 94:062304, 2016.

[22] C.S.Marians and L.W.Hobbs. J. Non-Cryst. Solids, 124:242, 1990.

[23] Le Roux S and Jund P. Comput. Mater. Sci., 50:1217, 2011.

[24] L.Lichtenstein, M.Heyde, , and H-J Freund. Phys. Rev. Lett., 109:106101, 2012.

[25] K.M.Burson, L.Gura, B.Kell, C. Buechner, A.L.Lewandowski, M.Heyde, and H-J Freund. Resolving amorphous solid-liquid interfaces by atomic force microscopy. Appl. Phys. Lett., 108:201602, 2016.

[26] P.Tangney and S.Scandolo. J. Chem. Phys., 117:8898, 2002.

[27] M.Wilson, P.A.Madden, M.Hemmati, and C.A.Angell. Phys. Rev. Lett., 77:4023, 1996.

[28] M.Wilson. Modelling networks in varying dimensions. In Molecular Dynamics Simulations of Disordered Materials, pages 215-254. Springer, 2015. 
[29] M. Wilson. Structure and dynamics in network-forming materials. Journal of Physics: Condensed Matter, 28(50):503001, 2016.

[30] M. Wilson. Structure, dynamics and multiple length-scales in network-forming materials. J. Stat. Mech., page 074010, 2016.

[31] A.W.Robertson, C.S.Allen, Y.Wu, K.He, J.Oliviera, J.Neethling, A.I.Kirkland, and J.H.Warner. Nature Comm., 3:1144, 2012.

[32] J.H.Warner, E.R.Margine, M.Mukai, A.W.Robertson, F.Giustino, and A.I.Kirkland. Science., $337: 209,1985$.

[33] A.Sartbaeva, S.A.Wells, M.M.J.Treacy, and M.F.Thorpe. The flexibility window in zeolites. Nature Materials, 5:962, 2006.

[34] H.S.M.Coexter. Regular Polytopes. Dover, 1973.

[35] D.Ormrod Morley and M.Wilson. Controlling disorder in two-dimensional networks. J. Phys.: Condens. Matter, page submitted, 2018.

[36] A.Malashevich, S.Ismail-Beigi, and E.I.Altman. Directing the structure of two-dimensional silica and silicates. J. Phys. Chem. C, 120:26770, 2016.

[37] S.N.Chiu. Mat. Char., 34:149, 1995.

[38] A.L.Lewandowski, P.Schlexer, C. Buechner, E.M.Davis, H.Burrall, K.M.Burson, W-D.Schneider, M.Heyde, G.Pacchioni, and H-J Freund. Atomic structure of a metal-supported two-dimensional germania film. Phys. Rev. B, 97:115406, 2018.

[39] C.J. Dawson, R. Sanchez-Smith, P. Rez, M. O'Keefe, and M.M.J. Treacy. Ab initio calculations of the energy dependence of $\mathrm{Si}-\mathrm{O}-\mathrm{Si}$ angles in silica and $\mathrm{Ge}-\mathrm{O}-\mathrm{Ge}$ angles in germania crystalline systems. Chemistry of Materials, 26(4):1523-1527, 2014.

[40] J.A.E. Desa. J. Non-Cryst. Solids, 51:57, 1982. 
(a)
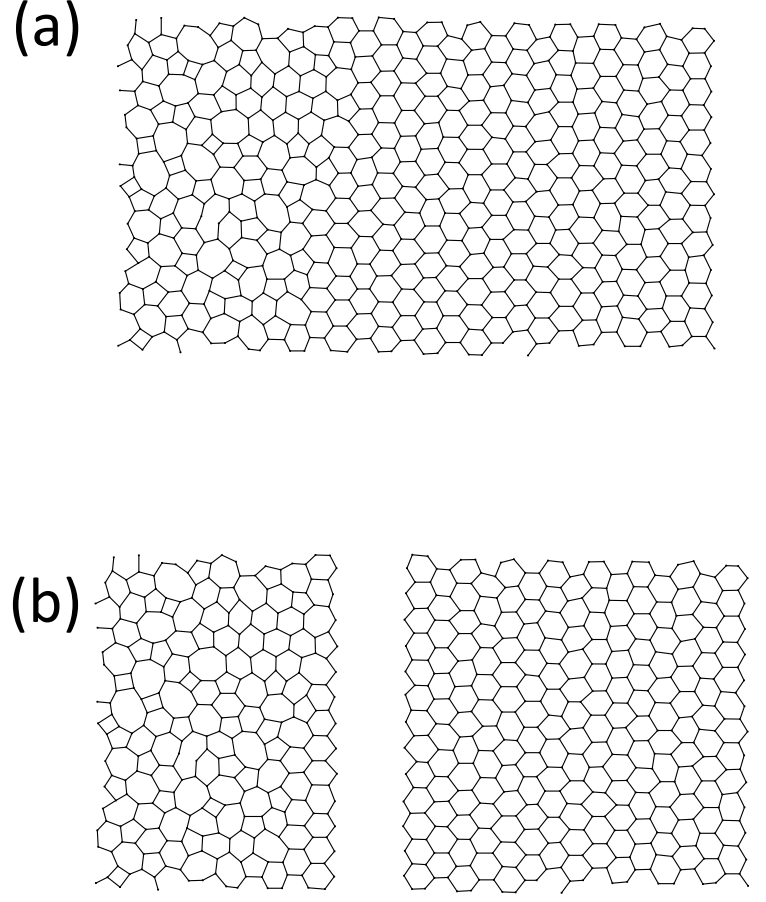

Figure 1. The upper panel shows the $\mathrm{SiO}_{2}$ bilayer configuration taken from reference [24] highlighting the Si atoms only. The configuration displays clear crystalline and amorphous regions. The configuration is separated as indicated in the lower panel to form crystalline and amorphous configurations. 

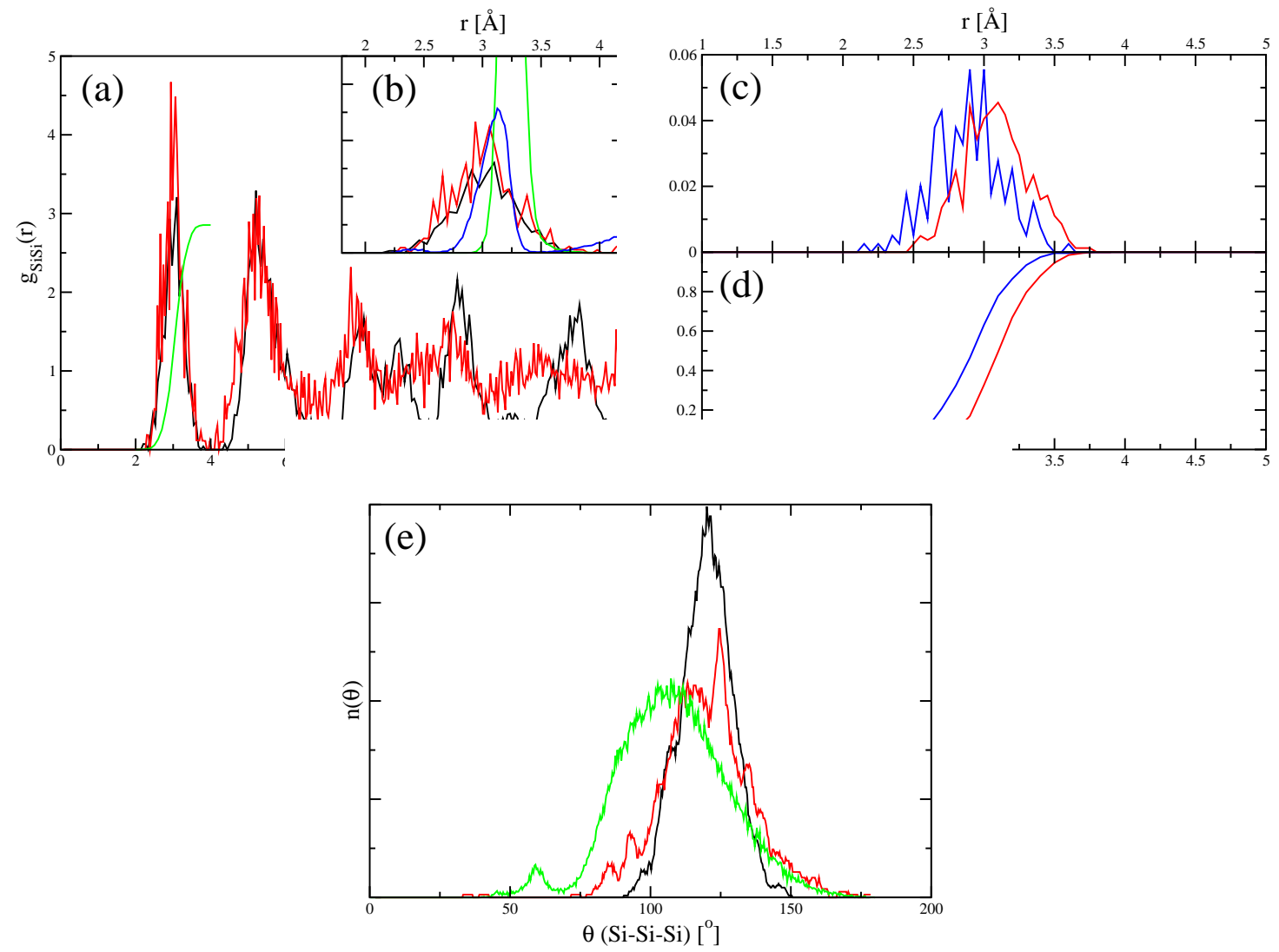

Figure 2. (a). Si-Si pair distribution functions, $g_{S i S i}(r)$, for the crystalline (black line) and amorphous (red line) regions (as shown in figure 1 from reference [24]). The green curve shows the cumulation coordination number distribution for the crystalline configuration. (b) The respective first peaks in $g_{S i S i}(r)$ compared to that obtained from three-dimensional amorphous configurations at ambient pressure (blue line - ref. [3]). The green line shows the Si-O distribution function, $g_{S i O}\left(r^{\prime}\right)$, with the length-scale doubled as described in the text. (c) Distribution and (d) cumulative distribution of the Si-Si nearest-neighbour separations obtained for $\mathrm{Si}-\mathrm{Si}$ pairs approximately perpendicular to the original interface (blue lines) and those at an angle of $\sim 30^{\circ}$ (red lines). (e) Si-Si-Si bond angle distributions generated from the amorphous (red line) and crystalline (black line) configurations (from ref. [24]) and compared to that obtained from three dimensional amorphous configurations (from ref. [3]) obtained at ambient pressure. 

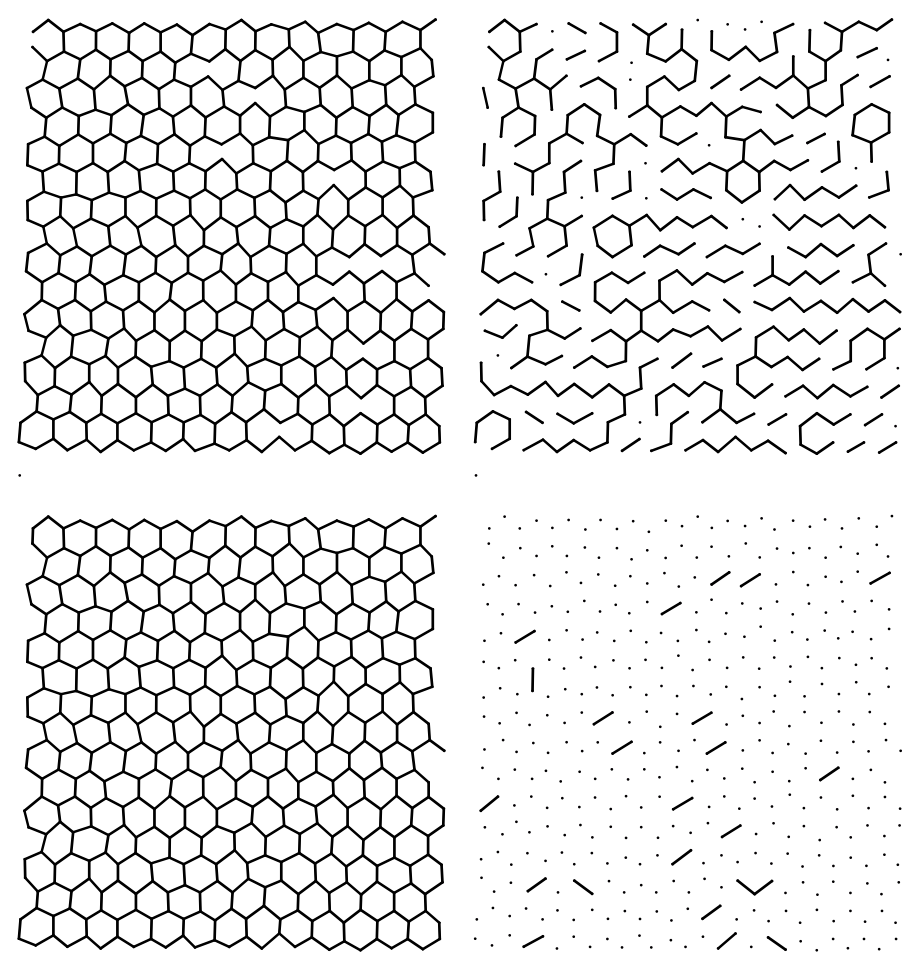

Figure 3. Molecular graphics "snapshots" for the Si atoms only from the Berlin group amorphous/crystal interface configuration [24] showing the crystalline section only. The figure shows bonds drawn between $\mathrm{Si}$ atoms with an upper "cut off" of $4.0 \AA$ and taking a successively longer lower bound. Key: bottom left - all bonds shown, bottom right - cut-off is $2.5 \AA$. top right - cut-off is $3.0 \AA$. top left - cut-off is $3.5 \AA$, 

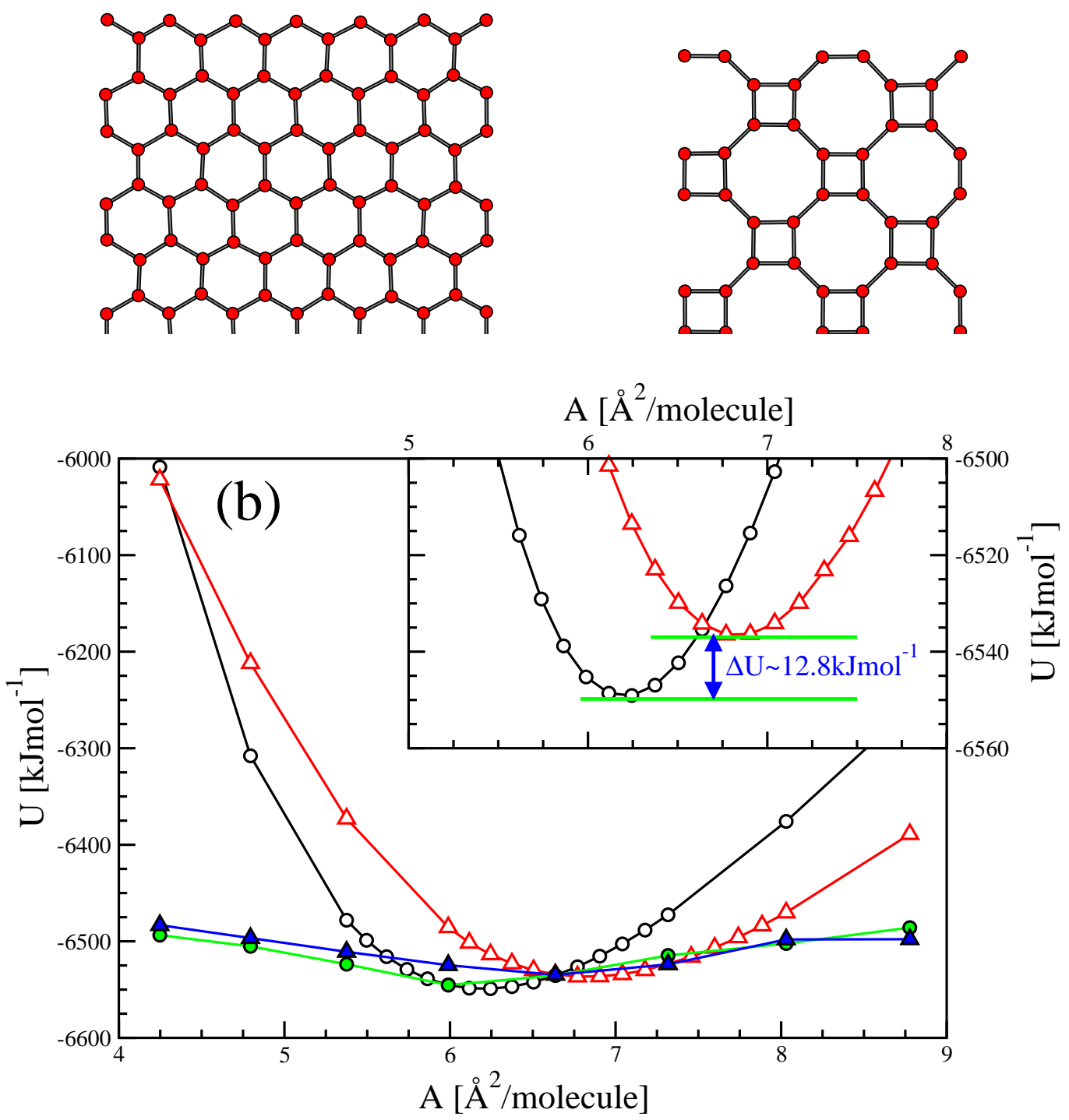

Figure 4. (a) Crystalline templates used to generate ideal bilayer crystals. Left: 6-membered rings only. Right: 4- and 8-membered rings. (b) Potential energy as a function of area per molecule (including both layers) for the relaxed bilayer structures (constructed from 6-membered rings [circles] and 4- and 8-membered rings [triangles] respectively). The open symbols refer to ideal structures relaxed (via steepest descent) to their local minima. The filled symbols refer to structures which have been through a heating/cooling cycle. The inset shows the ideal curves only in the region of their respective local minima. 
(a)
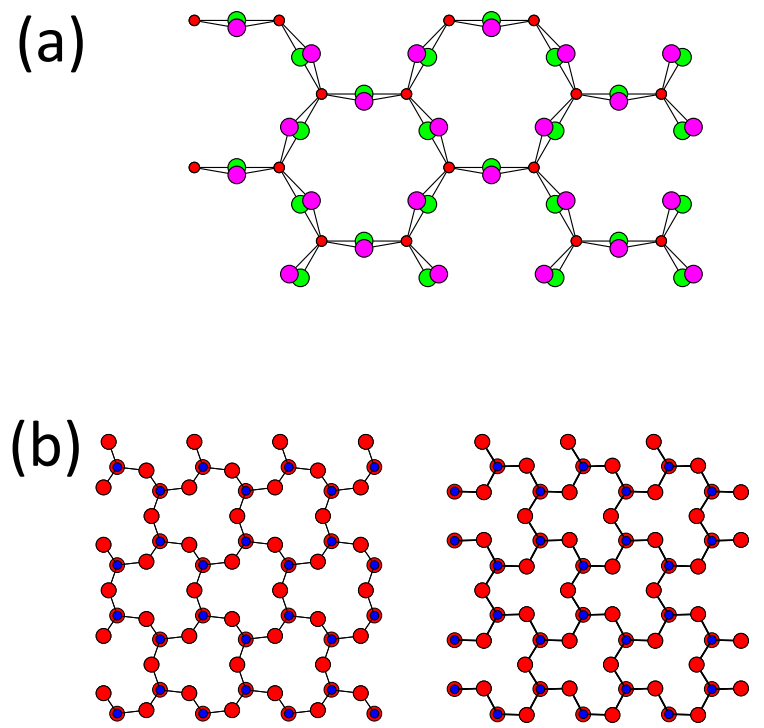

Figure 5. (a) Ideal distortions of the Si-O-Si triplets. The $\mathrm{O}$ atoms (large circles) are displaced along vectors perpendicular to the nearest-neighbour Si-Si "bonds". (b) Ideally distorted bilayers (left - small distortion, right - large distortion). In both cases the only distortions involve a coherent rotation of the tetrahedra about the inter-layer $\mathrm{Si}-\mathrm{O}-\mathrm{Si}$ vector. 


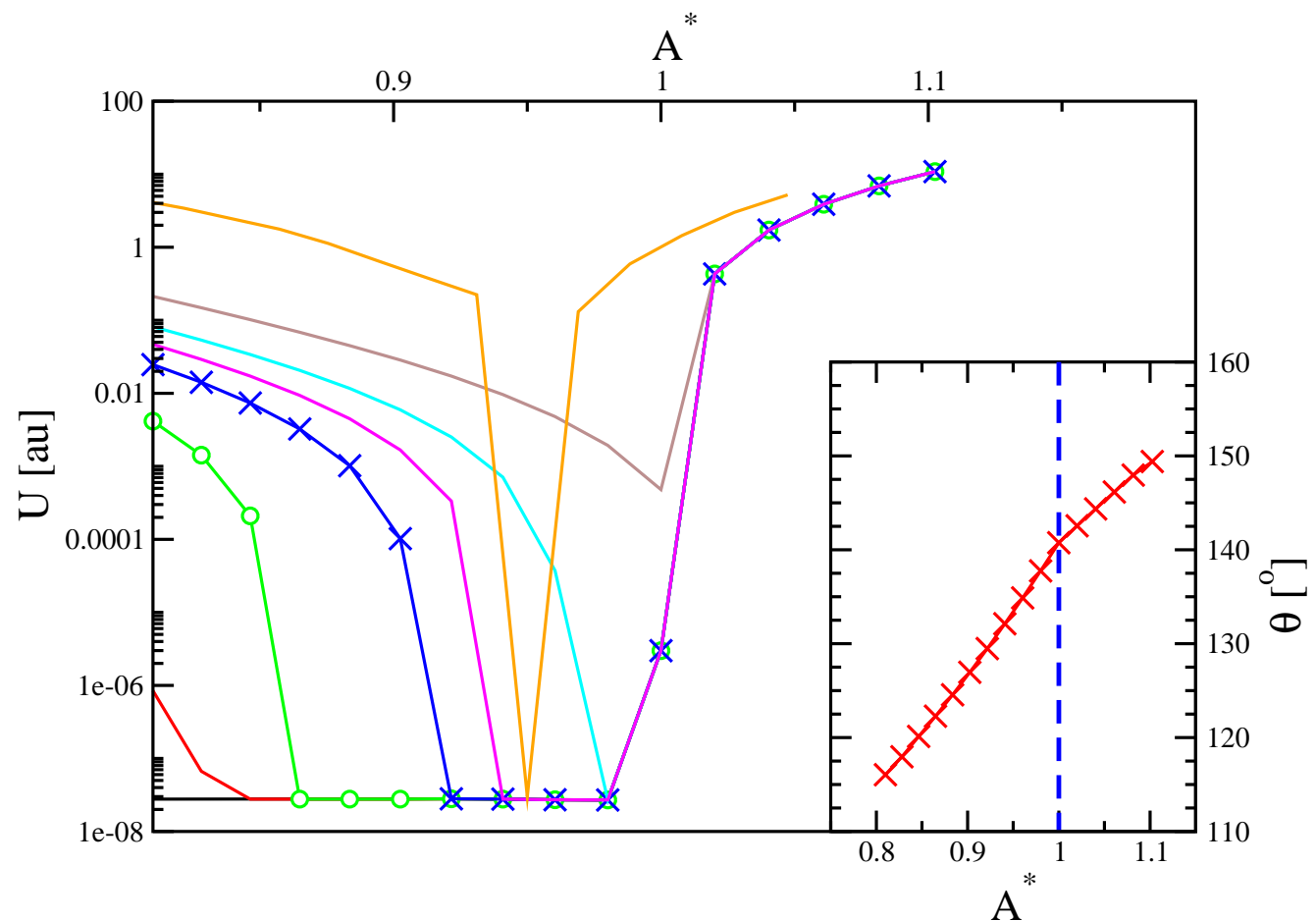

Figure 6. Energy $v$. area curves for the relaxed bilayer structures with distortions. The majority of curves are for the harmonic potential with varying inter-tetrahedral repulsion. Key: black line - no repulsion, red line $-\sigma=1.60$ green line $-\sigma=1.65$ blue line $-\sigma=1.70$ magenta line $-\sigma=1.725$ light blue line $-\sigma=1.75$ brown line $-\sigma=1.80$. The orange line shows the energy obtained using the TS potential (and which as the minimum energy subtracted). The inset shows the Si-O-Si bond angle as a function of the area with the dashed line highlighting the "transition" from the low area regime in which the energy can be driven to zero via coherent distortions, and the high area regime in which the tetrahedra become distorted. 
Crystalline Thin Films of Silica: Modelling, Structure and Energetics.
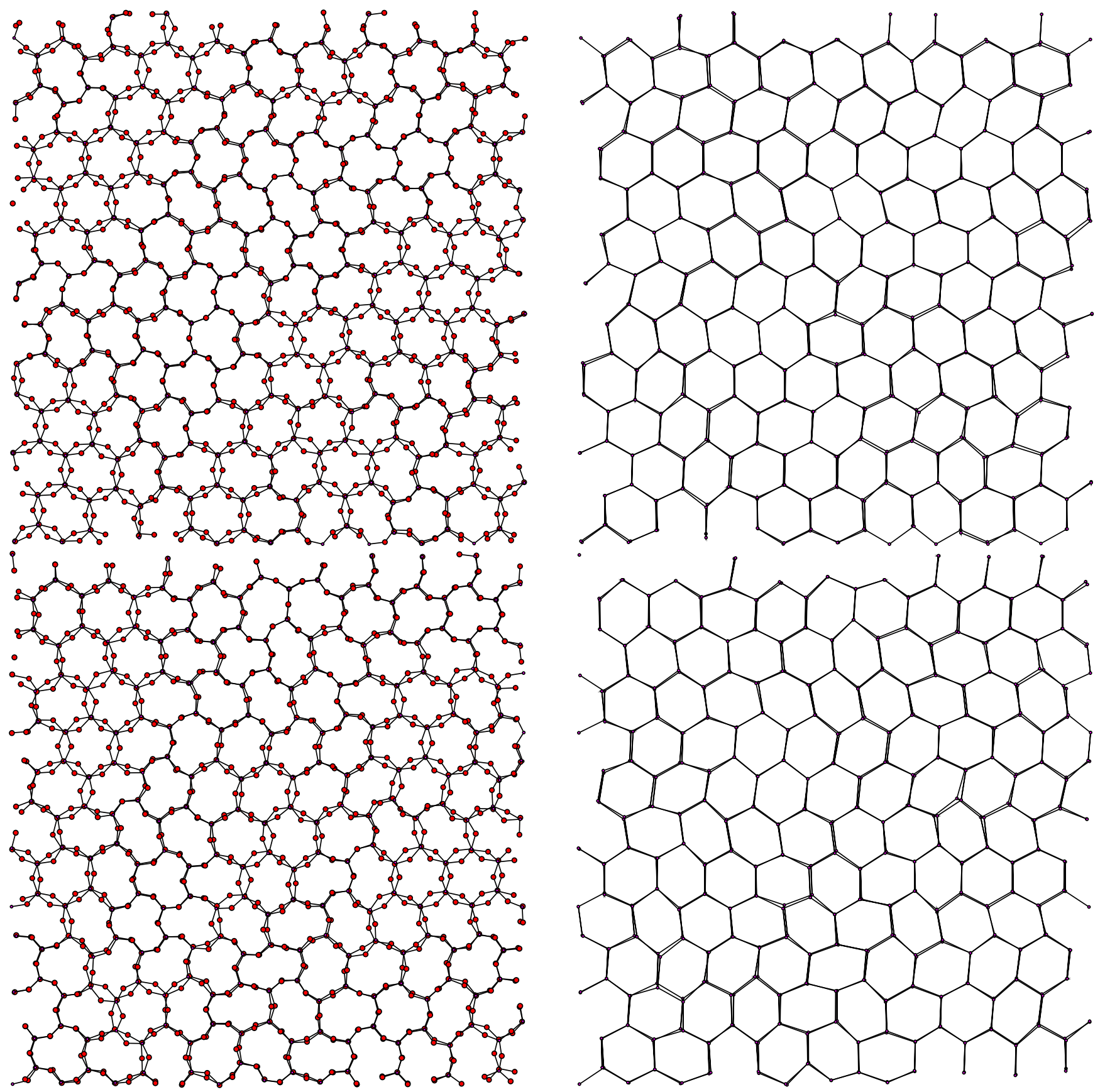

Figure 7. Distortions of the type highlighted in figure 5 for a bilayer heated and then cooled using the TS potential. The upper and lower figures show the results of two distinct cycles. In both cases the left panel shows the $\mathrm{Si}$ and $\mathrm{O}$ atoms (blue and red circles respectively) whilst the right panel shows the Si atoms only. 
Crvstalline Thin Films of Silica: Mr
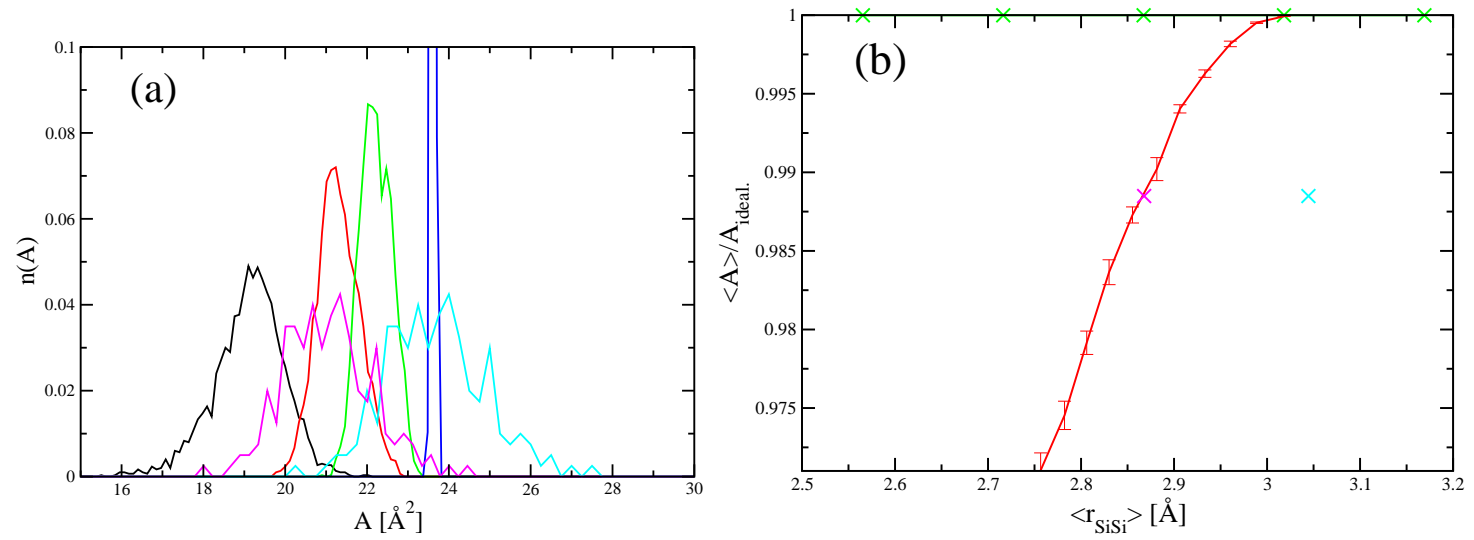

Figure 8. (a) Distributions of rings areas obtained from the TS potential at different areas and compared to that obtained from the experimental crystalline configuration. Key: (TS potential). Blue line $-A^{*}=0.95$, green line $-A^{*}=0.90$ red line $-A^{*}=0.86$ black line $-A^{*}=0.77$. Key (experimental configuration). Light blue line - raw data, magenta line - scaled by $\sim 0.94$ as described in the text. (b) The area per six-membered ring normalised by the ideal (undistorted) area as a function of the mean nearestneighbour Si-Si separation. The light blue cross shows the original experimental data point with the magenta cross showing the effect of the scaling as in panel (a). The red line shows the effect of randomly distorting the crystal using the TS potential whilst the green line shows the values obtained for the ideal distortion using the harmonic potential. 


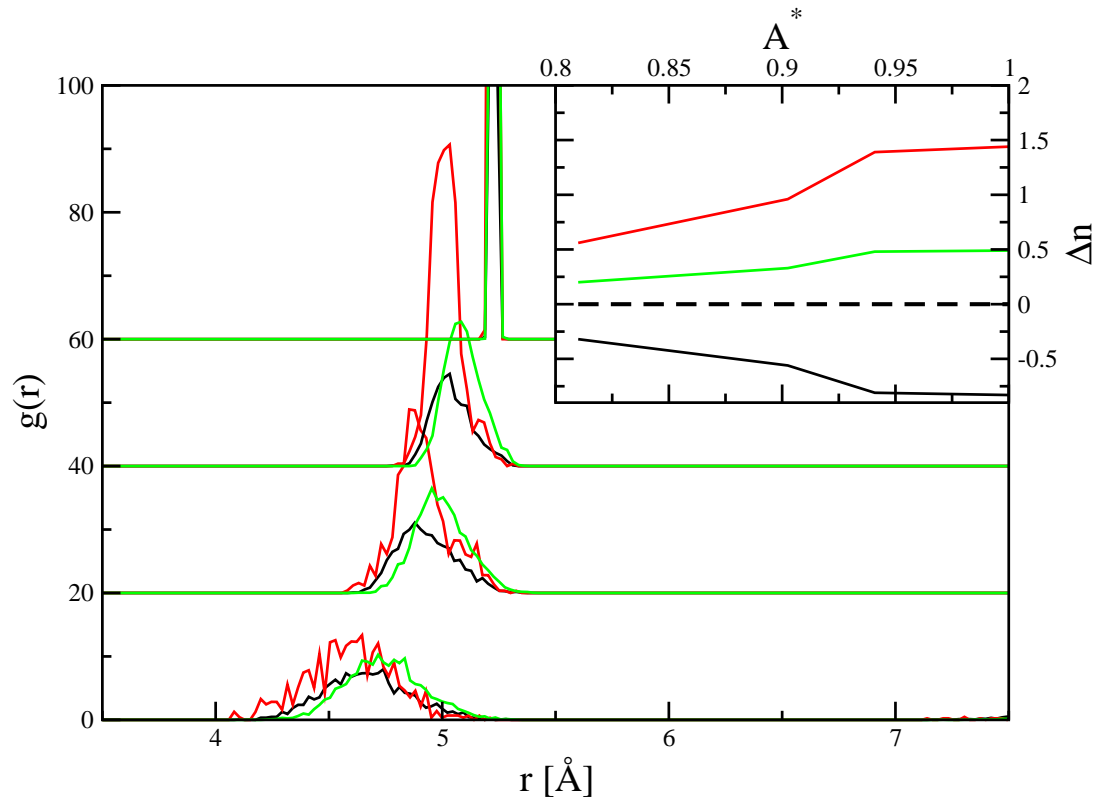

Figure 9. (Main panel) Radial distribution functions defined in terms of the centres of mass of the six-membered rings in the crystalline configurations and obtained by colouring the rings in terms of the distribution of areas (figure 8). Rings labelled "1" are from the lowest quartile of the distribution whilst those labelled "2" are from the remainder of the distribution. Key: red line $-g_{11}\left(r_{C M}\right)$, black line $-g_{12}\left(r_{C M}\right)$, green line $-g_{22}\left(r_{C M}\right)$. The effect of increasing the area is shown from bottom to top (displaced for clarity). The inset shows the difference (with respect to a random distribution) in nearest-neighbour ring-ring coordination numbers for the 1-1 (red line), 1-2 (black line) and 2-2 (green line) spatial correlations respectively as a function of area. 


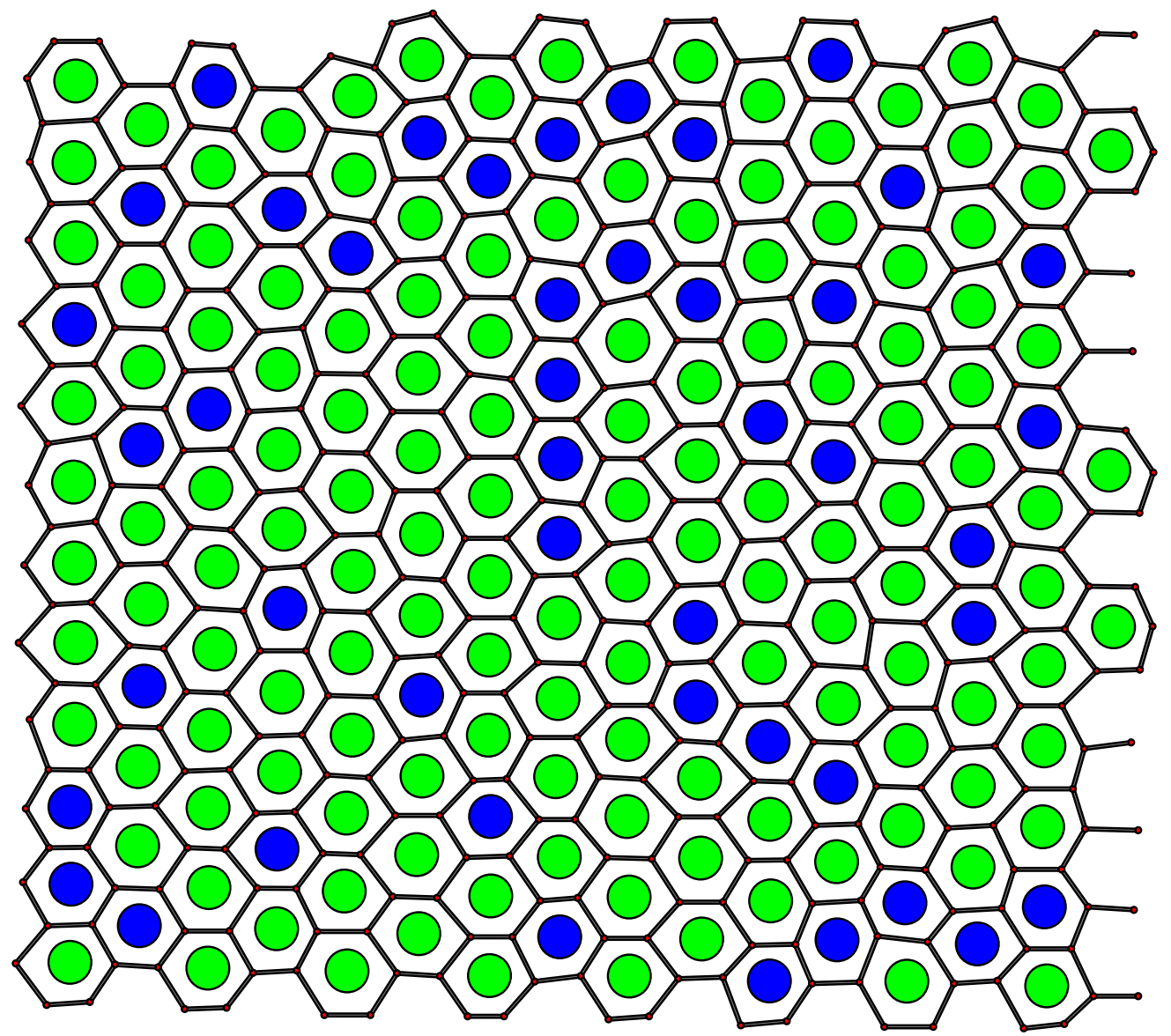

Figure 10. The experimental crystalline configuration (from ref. [24]) with the hexagons from the lowest quartile of the area distribution (figure 8) and the remainder shown as blue and green circles at the respective centres of mass. 